\title{
Peripubertal dietary flavonol and lignan intake and age at menarche in a longitudinal cohort of girls
}

\author{
Nancy A. Mervish ${ }^{1}$, Susan L. Teitelbaum ${ }^{1}$, Ashley Pajak ${ }^{1}$, Gayle C. Windham ${ }^{2}$, Susan M. Pinney ${ }^{3}$, Lawrence H. Kushi ${ }^{4}$, \\ Frank M. Biro ${ }^{5}$, Mary S. Wolff ${ }^{1}$ and on behalf of the Breast Cancer and Environment Research Programs
}

BACKGROUND: Dietary phytoestrogens may alter hormonal activity in childhood. Flavonols and lignans are the most prevalent phytoestrogens in the Western diet. We examined whether higher intake of flavonols and lignans was associated with later age at menarche in a prospective study of young girls.

METHODS: In all, 1,044 girls aged 6-8 years (mean 7.3 years) with two to four 24-h dietary recalls during their baseline year were followed up for 11 years until the attainment of menarche in the Breast Cancer and Environment Research Project (BCERP). Associations of age at menarche with quintiles of phytoestrogens were assessed using hazard ratios (HR) and 95\% confidence intervals (Cls) from Cox proportional hazards models, controlling for body mass index and other covariates.

RESULTS: The highest quintile of flavonol intake was associated with a later age at menarche, compared with the lowest quintile (adjusted HR: 0.80, 95\% Cl: (0.66-1.00). For lignans, there was a later age in overweight girls (HR: 0.56, 95\% $\mathrm{Cl}=0.40-0.80)$.

CONCLUSION: These dietary bioactives may reflect a healthy diet, and foods high in phytoestrogens may influence the timing of menarche.

E arly menarche has been associated with breast, endometrial, and ovarian cancer, possibly because it may lead to an increased lifetime estrogen exposure (1). Although timing of menarche is under genetic control (2), environmental factors may also have an important role. Childhood nutrition affects adolescent growth and pubertal development (3). In addition to the overall nutrition status, specific dietary components during childhood may influence the timing of puberty and menarche. Fiber $(4,5)$ has mainly been associated with a later age at menarche, whereas animal fats (6) and protein $(7)$ and dairy $(8,9)$ are associated with earlier menarche, although not consistently across studies (10-13). Longitudinal studies of phytoestrogens, including soy formula, dietary and urinary isoflavones, enterolactone, and flavonols, have shown later pubertal development (14-17). Reasons for differences may include prospective vs. crosssectional study design and timing of diet collection. Understanding the relationship between diet and menarche can help identify modifiable risk factors for disease prevention later in life.

Phytoestrogens are naturally occurring plant-derived polyphenols found in many foods that have been postulated to have beneficial health effects (18). The main groups of phytoestrogens are lignans, isoflavones, and coumestans. Numerous epidemiologic studies have suggested that dietary intake of phytoestrogens, particularly isoflavones, have a role in preventing certain types of hormonally dependent cancers, including breast and prostate cancers $(19,20)$. Mechanisms of the protective effect are not fully understood, although it may be explained by the competition of phytoestrogens with endogenous estradiol for hormone receptors or an inhibitory effect on aromatase activity and antioxidant properties (21). The intake of soy products that are the major contributors to isoflavone intake is low in Western societies, whereas the intake of Flavonols and lignans is higher in the Western diet. Flavonols and lignans have weak estrogenic activity (22) and they have been associated with estrogen-dependent outcomes, such as puberty, fecundity, menarche, and ovarian hormone profiles $(19,23,24)$.

Identifying the major sources of foods eaten that contain high concentrations of these micronutrients in young girls may allow for targeted recommendations for health-care providers. Our previous work has shown these phytoestrogens to be associated with a delay in girls' breast development (14); therefore, we hypothesize that they also may be associated with later menarche. We examined whether increased intake of flavonols and lignans before puberty is associated with later age at menarche in a prospective study of young girls.

\section{METHODS}

This project was a part of the NIEHS/NCI Breast Cancer and the Environment Research Project (BCERP). BCERP is an observational

\footnotetext{
${ }^{1}$ Department of Environmental Medicine and Public Health, Icahn School of Medicine at Mount Sinai School, New York, New York; ${ }^{2}$ California Department of Public Health, Division of Environmental and Occupational Disease Control, Richmond, California; ${ }^{3}$ Department of Environmental Health, University of Cincinnati College of Medicine, Cincinnati, Ohio; ${ }^{4}$ Division of Research, Kaiser Permanente Northern California, Oakland, California; ${ }^{5}$ Department of Pediatrics, Cincinnati Children's Hospital Medical Center, Cincinnati, Ohio. Correspondence: Nancy A. Mervish(nancy.mervish@mssm.edu) 


\section{Articles Mervish et al.}

longitudinal study of pubertal development of 1,239 girls enrolled at 6-8 years of age. Enrollment occurred between 2004 and 2007 at three sites as follows: Icahn School of Medicine at Mount Sinai (NYC), which recruited through clinics, schools, and neighborhood centers in East Harlem, NY, with girls seen annually; Cincinnati Children's Hospital/University of Cincinnati (Cincinnati, $\mathrm{OH}$ ), which recruited through schools in the Cincinnati metropolitan area and through the Breast Cancer Registry of Greater Cincinnati, with girls seen semi-annually; and Kaiser Permanente Northern California (KPNC), which recruited KPNC Health Plan members in the San Francisco Bay Area, with girls seen annually. The study was approved by the institutional review board at each site. Eligible girls had no underlying conditions affecting metabolism or growth. All sites obtained informed consent from parent or guardian and assent from girls. Parents or guardians of the participants identified girls' race/ethnicity as Black, White, and Asian, and ethnicity as Hispanic or non-Hispanic. At enrollment (baseline), and approximately annually for 11 years, parents or guardians were interviewed in person or were made to answer a self-completed questionnaire, in either English or Spanish, about the children's environmental exposures, physical activity, medical history, and demographics. Anthropometry and pubertal staging were assessed at each visit. Questions about the presence and date of first menarche were asked after the first follow-up year in NYC and KPNC and $\sim 5$ years after baseline in Cincinnati. A complete description of the study design is available in Biro et al 2010 (ref. 25). We report on 1,044 girls with two to four diet recalls provided within their first year of enrollment, who were asked about menarche at least once during 2014 (mean years of follow-up $=9$ ).

\section{Assessment of Age at Menarche}

Age at menarche was assigned based on questionnaire information provided primarily by the parent or guardian $(N=795,76 \%)$ or based on the girl's self-reported information $(N=112,11 \%)$. Girls who did not reach menarche were censored for statistical purposes at their age at their last visit or response $(n=137,13 \%)$.

\section{Dietary Data}

Dietary recall interviews were performed by trained dieticians at the Cincinnati Center for Nutritional Research and Analysis using the NDSR, Minn, Minnesota. Multiple (two to four) 24-h recalls by telephone were conducted with caregivers during the first year to provide an average intake over a year's time, as previously described (14). Average intakes over 1 year account for daily and seasonal variation in food consumed. Three recalls have been shown to reliably rank individuals on energy and most nutrients (26). The majority of girls had a complete set of four recalls $(N=984)$; the remainder had either two or three recalls $(N=148$ and 46 , respectively). Recalls with either extremely low or high total energy consumption values ( $<400$ and $>4,000 \mathrm{k} / \mathrm{cal}$ per day) were excluded from analysis $(N=18)$.

We developed a dietary database that captured flavonol and lignan contents of several hundred foods that were unavailable in standard dietary databases including NDSR (14). We assigned flavonol and lignan content $(\mathrm{mg} / 100 \mathrm{~g})$ for 2,319 individual foods and components reported, using published sources, including the United States Department of Agriculture (USDA). Flavonol and lignan intake was calculated for each reported food by multiplying the quantity consumed (g/day) and the phytoestrogen content (mg/g). Total daily flavonol and lignan intake (mg/day) was averaged over the two to four calls for each girl.

\section{Statistical Analyses}

Baseline characteristics of the study population were examined by quintiles of flavonol and lignan intake. Owing to skewed distributions, continuous variables are summarized as the medians and interquartile ranges. We tested for differences across quintiles of phytoestrogens by demographic characteristics using either $\chi^{2}$-test (categorical variables) or Kruskal-Wallis test (continuous variables).
We computed the amount of total flavonol and lignans in a standard serving size of foods selected as being high in these phytoestrogens, and then compiled the frequency of girls in our study who ate these foods (Tables 2 and 3 ).

Longitudinal analyses, examining associations between baseline flavonol and lignan intake and menarche, utilized reports of menarche over 11 years of annual follow-up visits. We used Cox proportional-hazards models to calculate the HRs and 95\% CIs for associations between phytoestrogen levels and menarche. Proportional hazards' assumptions were met in all of our analyses. Censoring occurred at the age of first menstrual period or at the age at last visit if menarche had not been reported. We examined flavonol and lignan intake using quintiles unadjusted and calorie adjusted using the nutrient density method to remove variation caused by total energy intake (26). Associations were similar, and only calorie-adjusted values are reported. All analyses were performed using the Statistics Analysis System (SAS) statistical software, Version 9.3 (SAS Institute, Cary, NC).

Confounding by potential covariates was assessed for the following variables: self-declared race/ethnicity, parent or guardian education, total daily average calories, fiber intake, and BMI percentile (BMI\%) dichotomized at the 85th percentile, which defines overweight (specific for age and gender) according to the CDC (27). We examined BMI\% separately at two time points-at baseline and at visit closest to menarche. These variables were chosen because they have been associated with both menarche and diet in the literature and because they were based on a conceptual model of diet and pubertal development. We included covariates that altered the estimate by more than $10 \%$. We did not include site because of its collinearity with race and BMI\%. Final models were adjusted for selfdeclared race/ethnicity and baseline BMI\%. Tests for trend across phytoestrogen quintiles were performed by including flavonol and lignan quintile ordinals as continuous variables. P-contrasts were obtained from the contrast statement comparing the HR of the first quintile to the fifth quintile. We obtained adjusted median pubertal ages for flavonol and lignan intake using survival function estimates from the baseline survivor function of multivariable-adjusted Cox models with quintiles as a strata statement. We evaluated race and baseline $\mathrm{BMI} \%$ as effect modifiers by adding interaction terms to the model (flavonol or lignan quintile $\times$ BMI\% dichotomized at the 85 th percentile and flavonol or lignan quintile $\times$ race). If there was a significant interaction with either race or BMI\% we examined stratum-specific models.

\section{RESULTS}

Table 1 presents the demographic characteristics of the study population at baseline. All girls consumed at least one food with flavonol and lignan content. The median intake of flavonols was $6.0 \mathrm{mg} /$ day and that of lignans was $0.40 \mathrm{mg} /$ day. Flavonols and lignans are found in lower concentrations in more frequently consumed foods such as potatoes, grains, cereals, tomato products, and coffee (Tables 2 and 3 ) compared with those in foods like kale and arugula. The average intake consumed in our population for both flavonol and lignans was a full serving per day for some of the foods (e.g., grapefruit juice, pears, and strawberries).

Tables 4 and 5 show the baseline characteristics of 1,044 participants according to quintiles of flavonol and lignan intake. Girls with higher flavonol and lignan intake were more likely to be white, have more educated parents/guardians, and consume more calories. On their last visit, 907 (87\%) of the girls had reported a first menstrual period. The unadjusted median age at menarche in our sample was 12.2 years (interquartile range: 11.4-13.1 years). This was the same as 
the median of the total cohort of 1,089 reporting age at menarche (median age 12.3 years (interquartile range: 11.413.2 years), which included girls without diet data.

The highest quintile of flavonol intake was associated with a later age at menarche, compared with the lowest quintile

Table 1. Baseline characteristics of 1,044 BCERP participants, 2004-2014

\begin{tabular}{lcc}
\hline Characteristic & Mean (Std) & \\
\hline Baseline age (years) & $7.3(0.68)$ & $\%$ \\
Child race/ethnicity & $N$ & 30 \\
Black & 316 & 30 \\
Hispanic & 316 & 34 \\
White & 357 & 5 \\
Asian & 55 &
\end{tabular}

Parent or guardian education ${ }^{\mathrm{b}}$

$\begin{array}{lll}\leqslant \text { High school } & 296 & 29 \\ >\text { High school } & 725 & 71\end{array}$

Baseline BMI percentile ${ }^{c}$

\begin{tabular}{lll}
$<50$ th & 349 & 33 \\
$50-85$ th & 359 & 34 \\
$\geqslant 85$ th & 336 & 32 \\
\hline
\end{tabular}

BCERP, Breast Cancer and Environment Research Project; Std, standard.

${ }^{b} n=1,021$ girls with all variables.

${ }^{\mathrm{C}} \mathrm{BMl}(\mathrm{kg} / \mathrm{mg} 2)$ specific for age and sex according to CDC growth data. (adjusted hazard ratio (HR): 0.80, 95\% confidence interval (CI): (0.66-1.00; Table 6). Compared with the lowest lignan intake (median $0.14 \mathrm{mg} /$ day), highest intake (median $0.88 \mathrm{mg} /$ day) was also suggestive of an association with later menarche (adjusted HR: 0.84, 95\% CI: (0.66-1.04; Table 6). We additionally adjusted for average fiber intake and estimates were unchanged (data not shown). The BMI-lignan interaction was significant in the test for effect modification $(P$ for interaction $=0.02$ ). As shown in Table 7 , the association with later menarche was restricted to girls with higher BMI (HR: $0.56,95 \% \mathrm{CI}=0.40-0.80$ ). There was no evidence of interaction by BMI with flavonols nor was there interaction of the association of lignans or flavonols and menarche by race (data not shown).

\section{DISCUSSION}

Our findings suggest that specific childhood dietary factors may influence the pubertal trajectory. In this longitudinal cohort, flavonol consumption was associated with later age at menarche, in keeping with our hypothesis. Similar relationships were observed in this cohort between increased intake of these phytoestrogens and later age at breast development, which typically precedes menarche by $2-3$ years. Association of lignans was restricted to overweight and obese girls, whereas flavonol associations were not modified by BMI. There was a 6-month delay in menarche among overweight girls with high lignan consumption compared with those with low lignan intake. To put this age difference in perspective, a time period of 6 months is the difference seen in black and

Table 2. Food items containing high flavonol content and frequency of consumption at baseline in 1,044 BCERP participants, $2004-2014$

\begin{tabular}{|c|c|c|c|c|}
\hline Food & Serving size ${ }^{a}$ & $\begin{array}{l}\text { Total flavonol }(\mathrm{mg}) \\
\text { serving }^{\mathrm{b}}\end{array}$ & $N(\%)$ any reported intake & $\begin{array}{l}\text { Average BCERP intake }(\mathrm{mg}) \text { of } \\
\text { flavonol per day }{ }^{c}\end{array}$ \\
\hline Arugula & 1 Cup & 158.8 & 0 & \\
\hline Kale & 1 Cup & 68.9 & 0 & \\
\hline Cranberries, raw & $1 / 2$ Cup & 21.96 & $19(2)$ & 3.5 \\
\hline Onions & $1 / 2$ Cup & 19.51 & $913(87)$ & 4.2 \\
\hline Spinach & 1 Cup & 18.02 & $114(11)$ & 1.5 \\
\hline Blueberries & $1 / 2$ Cup & 10.59 & $90(8)$ & 4.2 \\
\hline Pears & 1 Medium fruit & 4.81 & $113(10)$ & 5.8 \\
\hline Plums & 1 Medium fruit & 4.01 & $58(5)$ & 4.6 \\
\hline Apple w/ skin & 1 Medium fruit & 3.38 & $836(80)$ & 3.5 \\
\hline Cherries & $1 / 2$ Cup & 2.92 & $36(3)$ & 1.5 \\
\hline Tomato/tomato products & $1 / 2$ Cup & 2.88 & $939(90)$ & 1.7 \\
\hline Cocoa dry powder, unsweetened & 1 Tablespoon & 2.88 & $204(20)$ & 1.2 \\
\hline Tea & $8 \mathrm{fl} \mathrm{oz}$ & 0.22 & 194 (19) & 5.4 \\
\hline Grapes & 1/2 Cup & 2.10 & $296(28)$ & 2.3 \\
\hline Strawberries & 1/2 Cup & 1.69 & 251 (24) & 2.8 \\
\hline
\end{tabular}




\section{Articles | Mervish et al.}

Table 3. Food items containing high lignan content and frequency of consumption at baseline in 1,044 BCERP participants, 2004-2014

\begin{tabular}{|c|c|c|c|c|}
\hline Food & Serving size ${ }^{a}$ & $\begin{array}{l}\text { Total lignan } \\
(\mathrm{mg}) / \text { serving }^{\mathrm{b}}\end{array}$ & $\begin{array}{l}N(\%) \text { any reported } \\
\text { intake }\end{array}$ & $\begin{array}{l}\text { Average BCERP intake }(\mathrm{mg}) \text { of } \\
\text { lignan per dayc }\end{array}$ \\
\hline Spinach & 1 Cup & 0.76 & $114(11)$ & 0.05 \\
\hline Orange juice & 3/4 Cup & 0.52 & $592(57)$ & 0.53 \\
\hline Grapefruit juice & 3/4 Cup & 0.52 & $17(2)$ & 1.80 \\
\hline Eggplant & 1/2 Cup & 0.40 & 5 & 0.12 \\
\hline Squash & 1/2 Cup & 0.38 & $69(6)$ & 0.21 \\
\hline Yams/sweet potatoes & $1 / 2$ Cup & 0.30 & $41(4)$ & 0.43 \\
\hline Broccoli & $1 / 2$ Cup & 0.23 & $314(30)$ & 0.09 \\
\hline Hamburgers/tacos & 2-3 Ounces & 0.21 & $563(54)$ & 0.16 \\
\hline Cauliflower & 1/2 Cup & 0.10 & $66(5)$ & 0.07 \\
\hline Tomato/tomato products & 1/2 Cup & 0.06 & $939(90)$ & 0.1 \\
\hline Peanut butter & 2 Tablespoons & 0.05 & $375(36)$ & 0.06 \\
\hline Apples/applesauce & 1 Medium fruit & 0.05 & $836(80)$ & 0.05 \\
\hline Pears, raw & 1 Medium fruit & 0.05 & $113(10)$ & 0.24 \\
\hline
\end{tabular}

BCERP, Breast Cancer and Environment Research Project.

The major contribution of foods to total lignan intake—orange juice $35 \%$, strawberries $17 \%$, broccoli $8 \%$, carrots $8 \%$, potatoes $6 \%$, and meat $5 \%$.

a Serving sizes based on the USDA guidelines.

${ }^{\mathrm{b}}$ Total lignan values were derived from the nutrient database described in Methods.

${ }^{c}$ Average lignan intake includes only girls eating that food.

Table 4. Baseline characteristics of participants by flavonol intake in BCERP, 2004-2014

\begin{tabular}{|c|c|c|c|c|c|c|}
\hline \multirow[t]{3}{*}{ Characteristic } & \multicolumn{5}{|c|}{ Median (IQR) } & \multirow[t]{3}{*}{$P$-value } \\
\hline & \multirow[b]{2}{*}{$1(n=204)$} & \multicolumn{2}{|c|}{ Quintile of Flavonol Intake } & \multirow[b]{2}{*}{$4(n=206)$} & \multirow[b]{2}{*}{$5(n=202)$} & \\
\hline & & $2(n=204)$ & $3(n=203)$ & & & \\
\hline Flavonols (mg/day) & $2.7(1.9-3.1)$ & $4.4(3.9-4.8)$ & $6.0(5.6-6.4)$ & $7.9(7.4-8.4)$ & $11.1(10.0-13.4)$ & \\
\hline Baseline age (years) & $7.2(6.6-7.8)$ & $7.2(6.7-7.8)$ & $7.4(6.9-7.8)$ & $7.5(6.9-7.8)$ & $7.4(6.9-7.8)$ & 0.06 \\
\hline Calories (kcal/day) & $1,413(1,209-1,650)$ & $1,495(1,290-1,730)$ & $1,533(1,386-1,790)$ & $1,601(1,453-1,860)$ & $1,674(1,453-1,860)$ & 0.001 \\
\hline Hispanic & 32 & 32 & 32 & 32 & 25 & \\
\hline White & 33 & 28 & 34 & 35 & 42 & \\
\hline Asian & 5 & 4 & 6 & 8 & 3 & \\
\hline \multicolumn{7}{|c|}{ Baseline BMI Percentile ${ }^{c}$} \\
\hline$<50$ th & 35 & 33 & 32 & 31 & 36 & 0.41 \\
\hline $50-85$ th & 29 & 31 & 39 & 34 & 36 & \\
\hline$\geq 85$ th & 36 & 35 & 29 & 36 & 28 & \\
\hline
\end{tabular}

BCERP, Breast Cancer and Environment Research Project; IQR, interquartile range.

${ }^{a} X^{2}$-test for categorical variables and Kruskal-Wallis test for continuous variables.

${ }^{b} n=1,021$ girls with all variables.

${ }^{c} \mathrm{BMI}\left(\mathrm{kg} / \mathrm{mg}^{2}\right)$ specific for age and sex according to CDC growth data. 
white girls in a number of studies (28). This present study is the only one that has examined the association between these two phytoestrogens, which are widespread in the Western diet, and age at menarche. Previous longitudinal and crosssectional studies have looked at soy and other dietary components and age at menarche with mixed results, possibly

Table 5. Baseline characteristics of participants by lignan intake in BCERP 2004-2014

\begin{tabular}{|c|c|c|c|c|c|c|}
\hline \multirow[t]{3}{*}{ Characteristic } & \multicolumn{5}{|c|}{ Median (IQR) } & \multirow[t]{3}{*}{$P$-value ${ }^{a}$} \\
\hline & \multirow[b]{2}{*}{$1(n=202)$} & \multicolumn{2}{|c|}{ Quintile of lignan intake } & \multirow[b]{2}{*}{$4(n=205)$} & \multirow[b]{2}{*}{$5(n=204)$} & \\
\hline & & $2(n=201)$ & $3(n=208)$ & & & \\
\hline Baseline age (years) & $7.3(6.6-7.8)$ & $7.2(6.7-7.7)$ & $7.4(6.9-7.9)$ & $7.3(6.7-7.7)$ & $7.4(7.0-7.9)$ & 0.01 \\
\hline Calories (kcal/day) & $1,458(1,278-1,678)$ & $1,534(1,293-1,728)$ & $1,544(1,327-1,826)$ & $1,599(1,379-1,792)$ & $1,598(1,432-1,792)$ & 0.001 \\
\hline Hispanic & 35 & 26 & 29 & 29 & 36 & \\
\hline White & 31 & 36 & 35 & 33 & 37 & \\
\hline Asian & 5 & 7 & 5 & 6 & 5 & \\
\hline \multicolumn{7}{|c|}{ Baseline BMI percentile ${ }^{c}$} \\
\hline$<50$ th & 30 & 37 & 37 & 35 & 28 & 0.41 \\
\hline 50-85th & 35 & 31 & 34 & 34 & 36 & \\
\hline$\geqslant 85$ th & 35 & 32 & 29 & 31 & 36 & \\
\hline
\end{tabular}

BCERP, Breast Cancer and Environment Research Project; IQR, interquartile range.

${ }^{a} X^{2}$-test for categorical variables and Kruskal-Wallis for continuous variables.

${ }^{b} n=1,021$ girls with all variables.

${ }^{\mathrm{C}} \mathrm{BMI}\left(\mathrm{kg} / \mathrm{mg}^{2}\right)$ specific for age and gender according to CDC growth data.

Table 6. HRs and 95\% Cl from Cox proportional models for two dietary phytoestrogens in relation to menarche BCERP, 2004-2014

\begin{tabular}{|c|c|c|c|c|c|c|}
\hline & \multirow[t]{2}{*}{ Quintile } & \multicolumn{5}{|c|}{ HR $(95 \% \mathrm{Cl})$ for menarche lignan and flavonol intake } \\
\hline & & 1 & 2 & 3 & 4 & 5 \\
\hline \multirow[t]{3}{*}{ Flavonols } & Median (mg/day) & 2.7 & 4.4 & 6 & 7.9 & 11.1 \\
\hline & $N$ per quartile & 208 & 209 & 209 & 209 & 208 \\
\hline & $\mathrm{HR}^{\mathrm{a}}(\mathrm{Cl})$ & 1 (Ref) & $1.04(0.84-1.28)$ & $1.09(0.88-1.34)$ & $1.02(0.82-1.25)$ & $0.83(0.66-1.03)$ \\
\hline \multirow[t]{4}{*}{ Lignans } & Median (mg/day) & 0.14 & 0.26 & 0.39 & 0.56 & 0.88 \\
\hline & Range (mg/day) & $0.01-0.196$ & $0.197-0.314$ & $0.316-0.45$ & $0.45-0.69$ & $0.69-2.76$ \\
\hline & $N$ per quartile & 208 & 209 & 209 & 209 & 209 \\
\hline & $\mathrm{HR}^{\mathrm{a}}(\mathrm{Cl})$ & 1 (Ref) & $0.80(0.65-0.99)^{*}$ & $0.88(0.71-1.09)$ & $0.84(0.69-1.04)$ & $0.85(0.69-1.05)$ \\
\hline
\end{tabular}

$\mathrm{Cl}$, confidence interval; BCERP, Breast Cancer and Environment Research Project; HR, hazard ratio.

*P $<0.05$.

a HR (CI): nutrient density calorie intake; unadjusted model.

${ }^{\mathrm{b}} \mathrm{HR}(\mathrm{CI})$ : nutrient density calorie intake; adjusted for race, baseline BMI\% diochotomized $>$ or $<85 \%$.

cPredicted from survivor function of adjusted Cox models. 


\section{Articles $\mid$ Mervish et al.}

Table 7. Interaction* of lignan intake and BMI with age at menarche

\begin{tabular}{|c|c|c|c|c|c|c|c|}
\hline \multirow{2}{*}{$\begin{array}{l}\text { Lignan intake } \\
\text { Quintiles }\end{array}$} & \multirow[t]{2}{*}{ All } & \multicolumn{5}{|c|}{ HR (95\% Cl) adjusted for race/ethnicity } & \multirow{2}{*}{$\begin{array}{l}\text { Median age at menarche } \\
\text { (months) }\end{array}$} \\
\hline & & $N$ & $\begin{array}{l}\text { Normal weight, } \\
\text { BMI }<85 \%\end{array}$ & $\begin{array}{c}\text { Median age at } \\
\text { menarche (months) }\end{array}$ & $N$ & $\begin{array}{c}\text { Overweight, obese } \\
\text { BMI } \geqslant 85 \%\end{array}$ & \\
\hline 1 & 1.00 (ref) & 135 & 1.00 (ref) & 148 & 73 & 1.00 (ref) & 137 \\
\hline 2 & $0.81(0.66-1.00)$ & 148 & $0.93(0.72-1.2)$ & 153 & 62 & $0.66(0.45-0.95)$ & 142 \\
\hline 3 & $0.91(0.74-1.12)$ & 147 & $1.1(0.78-1.4)$ & 149 & 62 & $0.66(0.45-0.94)$ & 140 \\
\hline 4 & $0.86(0.70-1.06)$ & 144 & $1.0(0.78-1.3)$ & 151 & 65 & $0.63(0.44-0.91)$ & 142 \\
\hline 5 & $0.84(0.68-1.04)$ & 134 & $1.1(0.84-1.4)$ & 148 & 75 & $0.56(0.40-0.80)$ & 143 \\
\hline
\end{tabular}

due to the time when diet was assessed during childhood and the type of database used $(10,11,13,16)$.

Our findings are particularly noteworthy, given that flavonol and lignan intake in this cohort is relatively low compared with a few studies reporting these phytoestrogens in US adults $(29,30)$. Intake of phytoestrogens is usually lower in children, especially in an urban low socioeconomic status population (14). Flaxseeds are rich sources of lignans $(80 \mathrm{mg}$ per $1 \mathrm{oz}$ serving size), although they were not consumed in our cohort. In contrast, flavonols and lignans are found in much lower concentrations in many more frequently consumed foods such as potatoes, grains, cereals, strawberries, coffee, tea, and wine. Girls in this cohort consumed a serving size per day of some of the foods with the highest content of flavonol and lignan (Tables 2 and 3). Isoflavones, important phytoestrogens associated with reduced risk of breast cancer, and a later onset of puberty come from different types of foods compared with flavonols and lignans. Isoflavones are found in soy foods (soybeans, tofu, soy milk, and soy nuts) that are infrequently consumed in the United States compared with the foods high in flavonols and lignans. We did not examine these in our study. Although some foods high in lignans, flaxseeds, and spinach also contain isoflavones, the concentration of isoflavones in these foods is extremely low when compared with that in soybeans.

BMI has been shown to be a strong predictor of early menarche and early pubertal development (31). We saw that overweight/obesity modified the relationship between lignan intake and age at menarche. This finding is consistent with evidence of possible mechanisms that lignans regulate adipogenesis through the modulation of the estrogen receptor-signaling pathway in both animal and human studies (21), and that phytoestrogens activate the PPAR $\gamma$ receptor that controls many genes associated with fat and energy metabolism (32). Lignans are metabolized to enterolactone and enterodiol by gut microbiota in the colon, and a few human studies have shown that higher consumption of lignans was associated with lower overall fat mass (33). Enterolactone is also an aromatase inhibitor, which could reduce estrogen levels essential for pubertal changes.

Studies of menarche have measured diet at different time points of childhood, ranging from 1-3 years (34) to mid childhood (9 years; (ref. 8)) and later (9-14 years; (10)). Evidence is mixed as to when the critical period may be for diet to have an impact on the occurrence of menarche. Studies have shown associations between early-childhood diet and growth and pubertal development $(35,36)$. Birth may be a critical window for environmental exposures related to pubertal development, as it is a time when the reproductive tract is susceptible to changes in the developmental process. Soy intake during infancy altered the timing of menarche (17). Rapid weight gain in infancy and early childhood is a determinant of timing of pubertal development, and this reflects the importance of early "windows" during which nutrition can have long-term consequences for growth and development (37). However, later "windows of susceptibility" may have a different influence. For example, a protective effect of dietary phytoestrogen intake on breast cancer risk was observed for relatively high isoflavone consumption before puberty (18) and during adolescence (38). Phytoestrogen exposure may need to occur in a critical window long before the outcome to have an influence.

Strengths of this study are its prospective nature, comprehensive diet assessment, and reliable outcome. We measured diet when girls were 6-8 years of age, several years before menarche, strengthening conclusions of the observed relationship. Diet was assessed using an average of multiple 24-h dietary recalls, and we utilized an extensive database created to assess flavonol and lignan intake. The idea that dietary intake may change over time has been studied in children aged 9-18 years; dietary intake patterns that were assessed 5 years apart were stable over time (39). A main limitation is the method of assessing flavonol and lignan intake. Our database was developed using the currently known information of foods with flavonol and lignan content, which may not have had flavonol and lignan content of every food item reported in the Nutrition Data System for Research (NDSR, Minn, Minnesota) questionnaire. Another method of lignan exposure assessment is using urine biomarkers; however, biomarkers have temporality concerns. In our previous report of this cohort for diet and breast development, we showed lowmoderate correlations between urine enterolactone and lignan intake assessed through dietary records, and urinary enterolactone was associated with pubertal development $(14,25)$. 
We report an association between higher childhood consumption of flavonols and lignans and later age at menarche. These agents have known anti-hormonal and anti-obesogenic properties. It is possible that flavonols and lignans reflect a healthy diet, as they are derived mainly from fruits, vegetables, and fiber in our cohort. However, the associations remain after adjusting for fiber, which may be another surrogate for a healthy diet. Although it is difficult to disentangle the individual effects of these phytoestrogens from fruits, vegetables, and fiber, nonetheless they all may have a role in the observed pubertal delay. Our findings suggest that specific dietary bioactives in certain fruits and vegetables may have additional long-term benefits, and dietary modifications are of continued importance to young girls.

\section{ACKNOWLEDGMENTS}

We gratefully acknowledge our collaborators at the three medical centers involved in this research including Jessica Guiterrez, Rochelle Osborne, Lisa Boguski, Joel Forman, and Barbara Brenner (MSSM); Gayle Greenberg, Bob Bornschein (Cincinnati); and Robert Hiatt, Louise Greenspan, Julie Deardorff (Kaiser Permanent.

\section{STATEMENT OF FINANCIAL SUPPORT}

This publication was made possible by Avon Foundation (New York, NY), the Breast Cancer and the Environment Research Program (BCERP) award numbers U01ES012770, U01ES012771, U01ES012800, U01ES012801, U01ES019435, U01ES019453, U01ES019454, and U01ES019457 from the National Institute of Environmental Health Sciences (NIEHS), Research Triangle Park, North Carolina, and the National Cancer Institute (NCl), Rockville, MD, P01ES009584, P30ES023514, P30ES023515, and P30ES0060696 from NIEHS, and UL1RR024131, UL1RR026314, and UL1RR029887 from the National Cancer for Research Resources (NCRR), Bethesda, MD.

\section{DISCLAIMER}

Its contents are solely the responsibility of the authors and do not necessarily represent the official views of the NIEHS or NCl, the National Institutes of Health, the Centers for Disease Control and Prevention, Atlanta, Georgia, or the California Department of Public Health.

Disclosure: The authors declare no conflict of interest.

\section{REFERENCES}

1. Rosner B, Colditz GA, Willett WC. Reproductive risk factors in a prospective study of breast cancer: the Nurses' Health Study. Am J Epidemiol 1994;139:819-35.

2. Wehkalampi K, Silventoinen K, Kaprio J, et al. Genetic and environmental influences on pubertal timing assessed by height. Am J Hum Biol 2008;20:417-23.

3. Rogol AD, Clark PA, Roemmich JN. Growth and pubertal development in children and adolescents: effects of diet and physical activity. Am J Clin Nutr 2000;72:521S-8S.

4. Hughes RE, Jones E. Intake of dietary fibre and the age of menarche. Ann Hum Biol 1985;12:325-32.

5. Koo MM, Rohan TE, Jain M, McLaughlin JR, Corey PN. A cohort study of dietary fibre intake and menarche. Public Health Nutr 2002;5:353-60.

6. Merzenich $\mathrm{H}$, Boeing $\mathrm{H}$, Wahrendorf J. Dietary fat and sports activity as determinants for age at menarche. Am J Epidemiol 1993;138:217-24.

7. Gunther AL, Karaolis-Danckert N, Kroke A, Remer T, Buyken AE. Dietary protein intake throughout childhood is associated with the timing of puberty. J Nutr 2010;140:565-71.

8. Wiley AS. Milk intake and total dairy consumption: associations with early menarche in NHANES 1999-2004. PLoS ONE 2011;6:e14685.
9. Ramezani TF, Moslehi N, Asghari G, et al. Intake of dairy products, calcium, magnesium, and phosphorus in childhood and age at menarche in the Tehran Glucose and Lipid Study. PLoS ONE 2013;8:e57696.

10. Carwile JL, Willett WC, Wang M, et al. Milk consumption after age 9 years does not predict age at menarche. J Nutr 2015;145:1900-8.

11. Moisan J, Meyer F, Gingras S. Diet and age at menarche. Cancer Causes Control 1990;1:149-54.

12. Cheng G, Buyken AE, Shi L, et al. Beyond overweight: nutrition as an important lifestyle factor influencing timing of puberty. Nutr Rev 2012;70:133-52.

13. Segovia-Siapco G, Pribis P, Messina M, Oda K, Sabate J. Is soy intake related to age at onset of menarche? A cross-sectional study. Nutr J 2014;13:54.

14. Mervish NA, Gardiner EW, Galvez MP, et al. Dietary flavonol intake is associated with age of puberty in a longitudinal cohort of girls. Nutr Res 2013;33:534-42.

15. Wolff MS, Britton JA, Boguski L, et al. Environmental exposures and puberty in inner-city girls. Environ Res 2008;107:393-400.

16. Cheng G, Remer T, Prinz-Langenohl R, et al. Relation of isoflavones and fiber intake in childhood to the timing of puberty. Am J Clin Nutr 2010;92:556-64.

17. Adgent MA, Daniels JL, Rogan WJ, et al. Early-life soy exposure and age at menarche. Paediatr Perinat Epidemiol 2012;26:163-75.

18. Adlercreutz H. Phytoestrogens and breast cancer. J Steroid Biochem Mol Biol 2002;83:113-8.

19. Buck K, Zaineddin AK, Vrieling A, Linseisen J, Chang-Claude J. Meta-analyses of lignans and enterolignans in relation to breast cancer risk. Am J Clin Nutr 2010;92:141-53.

20. Giles GG. Epidemiological investigation of prostate cancer. Methods Mol Med 2003;81:1-19.

21. Adlercreutz H, Bannwart $\mathrm{C}$, Wahala $\mathrm{K}$, et al. Inhibition of human aromatase by mammalian lignans and isoflavonoid phytoestrogens in childhood and age at menarche in the Tehran Lipid and Glucose Study. J Steroid Biochem Mol Biol 1993;44:147-53.

22. Setchell KD. Phytoestrogens: the biochemistry, physiology, and implications for human health of soy isoflavones. Am J Clin Nutr 1998;68:1333S-46S.

23. Linseisen J, Piller R, Hermann S, Chang-Claude J. Dietary phytoestrogen intake and premenopausal breast cancer risk in a German casecontrol study. Int J Cancer 2004;110:284-90.

24. Mumford SL, Sundaram R, Schisterman EF, et al. Higher urinary lignan concentrations in women but not men are positively associated with shorter time to pregnancy. J Nutr 2014;144:352-8.

25. Biro FM, Galvez MP, Greenspan LC, et al. Pubertal Assessment Method and Baseline Characteristics in a Mixed Longitudinal Study of Girls. Pediatrics 2010;126:e583-90.

26. Willett W, Stampfer MJ. Total energy intake: implications for epidemiologic analyses. Am J Epidemiol 1986;124:17-27.

27. CDC. Overweight and obesity 2015. Available at www.cdc.gov/obesity/ childhood/defining.html.

28. Euling SY, Selevan SG, Pescovitz OH, Skakkebaek NE. Role of environmental factors in the timing of puberty. Pediatrics 2008;121 (Suppl 3):S167-71.

29. Sampson L, Rimm E, Hollman PC, de Vries JH, Katan MB. Flavonol and flavone intakes in US health professionals. J Am Diet Assoc 2002;102: 1414-20.

30. Fink BN, Steck SE, Wolff MS, Kabat GC, Gammon MD. Construction of a flavonoid database for assessing intake in a population-based sample of women on Long Island, New York. Nutr Cancer 2006;56:57-66.

31. Biro FM, Khoury P, Morrison JA. Influence of obesity on timing of puberty. Int J Androl 2006;29:272-7.

32. Jungbauer A, Medjakovic S. Phytoestrogens and the metabolic syndrome. J Steroid Biochem Mol Biol 2014;139:277-89.

33. Hu Y, Song Y, Franke AA, Hu FB, van Dam RM. A prospective investigation of the association between urinary excretion of dietary lignan metabolites and weight change in US women. Am J Epidemiol 2015;182:503-11. 


\section{Articles | Mervish et al.}

34. Berkey CS, Gardner JD, Frazier AL, Colditz GA. Relation of childhood diet and body size to menarche and adolescent growth in girls. Am J Epidemiol 2000;152:446-52.

35. dos Santos Silva I, De Stavola BL, Mann V, Kuh D, Hardy R, Wadsworth ME. Prenatal factors, childhood growth trajectories and age at menarche. Int J Epidemiol 2002;31:405-12.

36. Sloboda DM, Hart R, Doherty DA, Pennell CE, Hickey M. Age at menarche: Influences of prenatal and postnatal growth. J Clin Endocrinol Metab 2007;92:46-50.
37. Ong KK, Ahmed ML, Emmett PM, Preece MA, Dunger DB. Association between postnatal catch-up growth and obesity in childhood: prospective cohort study. Br Med J 2000;320:967-71.

38. Thanos J, Cotterchio M, Boucher BA, Kreiger N, Thompson LU. Adolescent dietary phytoestrogen intake and breast cancer risk (Canada). Cancer Causes Control 2006;17:1253-61.

39. Cutler GJ, Flood A, Hannan P, Neumark-Sztainer D. Major patterns of dietary intake in adolescents and their stability. J Nutr 2009;139: $323-8$. 